\title{
La reformulación discursiva mediante es decir en el corpus PRESEEA de Granada
}

\author{
Natalia Ruiz-González ${ }^{1}$ \\ Universidad de Granada, España
}

\begin{abstract}
Resumen
Es decir es, para muchos lingüistas, uno de los marcadores que mejor reflejan la reformulación parafrástica en el discurso, esto es, la presentación de elementos equivalentes a otros enunciados emitidos con anterioridad pero que el hablante vuelve a formular, pues cree que han podido no quedar del todo claros. Para conocer el funcionamiento pragmático de esta partícula en la oralidad, hemos analizado su frecuencia de uso en el español de Granada, en el marco del corpus PRESEEA, y hemos estudiado qué sentidos contextuales toma y cómo se comporta en función de las variables sociales de los hablantes de la muestra. Los resultados han arrojado que su uso va desde la paráfrasis total hasta la recapitulación y la corrección, y que son los hablantes de nivel alto quienes prefieren esta forma por entenderla más formal y culta, así como que su uso predomina en los hombres y en la segunda generación, siendo este grupo el inmerso en el mercado laboral y el que debe asegurar su buena presencia
\end{abstract}

1 Para correspondencia, dirigirse a: Natalia Ruiz González (nataliaruiz@ugr.es), Departamento de Lengua Española, Facultad de Filosofía y Letras, Campus de la Cartuja, calle Profesor Clavera s/n, 18071, Granada. ORCID 0000-0002-3496-6933. 
ante los demás, por lo que se abandonan formas más coloquiales o estigmatizadas, como vamos u o sea.

Palabras clave: reformulación, explicativos: es decir, PRESEEA, sociolingüística, Granada.

\title{
DISCURSIVE REFORMULATION THROUGH ES DECIR IN THE PRESEEA CORPUS OF GRANADA
}

\begin{abstract}
Es decir is, for many linguists, one of the markers that best reflect the paraphrastic reformulation in the discourse, that is, the presentation of elements equivalent to other statements previously issued but that the speaker reformulates because he believes that they may not have been left completely clear. To know the pragmatic functioning of this particle in orality, we have analyzed its frequency of use in the Spanish of Granada, within the framework of the PRESEEA corpus, and we have studied what contextual senses it takes and how it behaves according to the social variables of the Sample speakers. The results have shown that its use ranges from total paraphrase to recapitulation and correction, and that it is high-level speakers who prefer this way to understand it more formal and cultured, as well as its use predominates in men and women. second generation, being this group the one immersed in the labor market and the one that must ensure its good presence before the others, so that more colloquial or stigmatized forms are abandoned, as vamos or o sea.
\end{abstract}

Keywords: reformulation, explicatives: es decir, PRESEEA, sociolinguistics, Grenade.

Recibido: $24 / 03 / 20$

Aceptado: $14 / 01 / 21$ 


\section{INTRODUCCIÓN ${ }^{2}$}

Hoy en día, el auge de los estudios sobre marcadores del discurso es incuestionable, especialmente los que versan sobre su definición y clasificación, pero resulta insuficiente aún el análisis de su distribución social en el discurso oral, y más en una lengua tan vasta y extensa como la española. Se deduce, por tanto, un especial interés averiguar cómo se organizan diatópica y diastráticamente estas unidades. El objetivo principal de este estudio es, pues, analizar el empleo de la partícula es decir en los hablantes de la ciudad de Granada.

Siguiendo la división de Martín Zorraquino y Portolés Lázaro (1999) y Portolés Lázaro (2014 [1998]), desde la Teoría de la Relevancia y la Teoría de la Argumentación, este marcador discursivo se inserta dentro de los llamados reformuladores explicativos, cuya función principal es volver sobre una parte previa del discurso para aclararla y matizarla por considerarla el hablante poco comprensible o ajustada a su intencionalidad comunicativa. El elemento reformulado es el que el oyente habrá de tomar en consideración a la hora de interpretar el acto comunicativo. Esa capacidad de reflexión sobre lo que el propio hablante produce viene, precisamente, condicionada por la dimensión metapragmática que los individuos tenemos sobre nuestro uso del lenguaje (Portolés Lázaro 2004: 37).

Para conocer el comportamiento pragmático y la distribución social de es decir en esta ciudad andaluza, utilizaremos el corpus PRESEEA de Granada y adoptaremos la idea de que toda elección de un marcador, como la de cualquier otro fenómeno lingüístico, puede estar condicionada por la pertenencia del hablante a un determinado sociolecto (Cortés Rodríguez 2008[1998]).

Partimos de la hipótesis de que esta unidad es empleada más frecuentemente en Granada por los hablantes de nivel socioeducativo alto, como ocurre en otras ciudades hispanohablantes (San Martín Núñez 2014: 311 ), mientras que los de nivel instruccional bajo preferirían otro tipo de unidades o procedimientos para llevar a cabo la función reformuladora, como vamos o vaya (Ruiz-González 2019). Además, consideramos que es una

\footnotetext{
2 Este trabajo ha sido financiado por el proyecto AGENDA 2050. El español de Granada: procesos de variación y cambio espaciales y sociales, con referencia PID2019-104982GB-C53, concedido por el Ministerio de Ciencia. Además, la autora es beneficiaria de un contrato predoctoral a través de la ayuda de formación de profesorado universitario del Ministerio de Universidades (FPU15-05294).
} 
partícula usada preferentemente por hablantes mayores, pues entendemos que los jóvenes pueden percibirla como una unidad excesivamente formal (Ciapuscio 2001: 169; Montolío Durán 2018: 504; San Martín Núñez 2014: 308).

En definitiva, en nuestro análisis buscaremos, por un lado, identificar la frecuencia de uso de este marcador, estudiar su comportamiento y los efectos de sentido que vehicula en la conversación, y, por otro lado, comprobar la relación entre su empleo y las variables sociolingüísticas de edad, sexo y nivel de instrucción.

\section{MARCO TEÓRICO}

Seguiremos para este trabajo los conceptos teóricos y metodológicos de la sociolingüística variacionista (Labov 1983), que percibe la variabilidad y heterogeneidad como rasgos inherentes de la lengua, aunque no aparezcan de manera aleatoria, sino reglada (Silva-Corvalán y Enrique-Arias 2017 [2001]: 268). La variación lingüística, así entendida, vendría condicionada por factores internos y externos al lenguaje, tales como el sexo, la edad, la etnia o el nivel sociocultural de los hablantes (Moreno Fernández 2015 [1998]). Asimismo, aceptamos que este tipo de variación puede aplicarse a cualquier nivel de la lengua y no solo al plano fonético; por ello, vamos a analizar unidades del plano pragmático-discursivo que, a pesar de su polifuncionalidad (Poblete Bennet 1997), pueden analizarse cuantitativamente si consideramos que dos o más partículas tienen equivalencia semántico-pragmática en determinados contextos y suponen interpretarse como dos formas de comunicar lo mismo (Cortés Rodríguez 2008 [1998]; Montoro del Arco 2011).

Partiremos para ello de otros trabajos que toman en cuenta la distribución espacial y social de los marcadores del discurso desde el punto de vista sociolingüístico, especialmente los citados en Carbonero Cano y Santana Marrero (2010), así como los estudios sobre marcadores integrados en el Proyecto de la Norma Culta Hispánica, como los monográficos editados por Valencia Espinoza (2014) y Valencia Espinoza y Vigueras Ávila (2015); los que se centran en grupos de marcadores específicos, como luego y después o los marcadores de interacción en la ciudad de Sevilla (Santana Marrero (2015a, 2016, 2017) y los que hacen un estudio general en ciudades específicas, como los de Sevilla también (Santana Marrero 2015b), 
Montevideo (Taibo Cao 2016), La Habana (González Mafud y Perdomo Carmona 2018) y Buenos Aires y Sevilla (Santana Marrero y Borzi 2020). Asimismo, nos interesan especialmente aquellos trabajos efectuados sobre otras ciudades andaluzas (Repede 2020; Santana Marrero 2014, $2015 \mathrm{c}^{4}$, 2016, 2019a, 2019b), así como los que ya se han desarrollado en Granada (Montoro del Arco 2011, 2012; Jaskot 2012). Además, de forma más específica pondremos la atención en aquellos que investigan las partículas de reformulación (Guirado Zapata 2019; San Martín Núñez 2014, 2016, 2017; San Martín Núñez y Guerrero González 2016), especialmente sobre nuestro corpus (Ruiz-González 2018, 2019, 2020).

\section{ESTADO DE LA CUESTIÓN}

Según el DLE (RAE 2020 s.v. es decir), es decir sirve para "dar a entender que se va a explicar mejor o de otro modo lo que ya se ha expresado", con lo cual este marcador tiene una clara función aclarativa con respecto al discurso anterior, sobre el que se pretende decir lo mismo, pero con otras palabras, para que quede lo más claro posible (Holgado Lage 2017). Además, el nivel de equivalencia es lo suficientemente alto para considerar el primer miembro y el segundo como dos formas exactas de expresar lo mismo. De hecho, algunos autores lo consideran el marcador de reformulación parafrástica por excelencia (Ciapuscio 2001: 169), incluso entre enunciados con una relación semántica débil (Bach Martorell 2009: 38-39).

Aunque llegó a ser considerado un galicismo de poco interés (Casado 1991), la Real Academia lo considera hoy sinónimo de o sea, como unidades equivalentes. Sin embargo, hay autores que perciben cierta especialización en el caso de $o$ sea, sobre todo por su uso reiterado en la oralidad, que lo lleva a tomar valores que es decir no tiene (Fuentes Rodríguez 1990, 2009).

Para López Alonso (1990: 94), esta unidad subordina el primer elemento reformulado al segundo, miembro reformulador en el que el marcador, por

\footnotetext{
3 Esta referencia se incluye en la monografía editada por Valencia Espinoza (2014), pero nos ha parecido conveniente señalarla de forma independiente por su comparabilidad con la ciudad de Granada.

4 Aquí ocurre lo mismo que en la nota anterior con respecto a la obra de Valencia Espinoza y Vigueras Ávila (2015).
} 
otra parte, se integra totalmente; y no es puramente parafrástico, sino que el segundo miembro incluye tanto lo dicho con anterioridad como los matices que se añaden a posteriori. En palabras de la propia autora, "el primer segmento propone como argumento una 'semántica interpretativa' y el segundo segmento, gracias al conector de reformulación 'es decir' explícita que se trata de una teoría semántica unificada" (López Alonso 1990: 94).

Y, aunque rara vez se ha hablado de es decir más allá de un valor puramente explicativo o parafrástico, algunos investigadores apuntan a la posibilidad de emplearlo para introducir una interpretación distinta o coorientada del elemento precedente (Beinhauer 1978 [1963]: 71, Galán Rodríguez 1998: 86), especialmente cuando este se encuentra implícito en la conversación (López Alonso 1990: 97), aunque, por lo general, no aparece presente ante miembros elididos, sino que su aparición viene condicionada por la explicitación del discurso previo. No obstante, a diferencia de otras unidades de su paradigma, sí que puede aparecer en posición inicial absoluta como punto de unión con elementos precedentes emitidos por el mismo hablante o por su interlocutor.

Igualmente, puede tomar un valor de soporte conversacional (Vigara Tauste 1992: 248), especialmente en hablantes de nivel alto, cuando se hace un uso excesivo de ella (Ciapuscio 2001, Fuentes Rodríguez 2009); también puede presentar un valor rectificativo (López Alonso 1990, Casado Velarde 1991, Santos Río 2003), especialmente en secuencias que incluyen magnitudes, (Ciapuscio 2001: 168) o, incluso, de distanciamiento (Ciapuscio 2001: 169).

Además, el hablante introduce "apreciaciones subjetivas" sobre la equivalencia semántica entre el elemento reformulado y el miembro reformulador, aportando matices y sentidos nuevos que limitan o acotan la interpretación del enunciado, para comportarse, en algunos casos, como un marcador de modalidad deóntica (Galán Rodríguez 1998: 98-99), tal como bueno, que también se usa como reformulador (Briz Gómez 1997: 72). La relación quedaría establecida como una afirmación en el miembro reformulado y su posterior justificación en el reformulador, equivalentes en tanto en cuanto el hablante utiliza matices que denotan esa relación (Galán Rodríguez 1998: 99).

En cuanto a su combinación con elementos de su entorno, "ante proposiciones o elementos que las resumen, frecuentemente engloba también la forma que, pero separada por pausa, como si perteneciera a la proposición que sigue, que no podrá ser interpretativa o asimilable" (Fuentes Rodríguez 2009), aunque en ocasiones entre ambos no se produce tal pausa y el que forma parte directa del marcador (Casado Velarde 1991: 107). La conjunción con frecuencia expresa un matiz de consecuencia de lo expresado, similar 
al que se apunta en o sea (Rodríguez Ramalle 2014, Murillo Ornat 2016, Ruiz-González 2018). Ocurre igual con otros explicativos que constituyen en algunos casos marcadores capaces de introducir conclusiones implicadas (Rodríguez Ramalle 2014: 126).

Con relación a su gramaticalización, parecen coincidir algunos autores en la ligera conservación de los términos que integran la unidad (ser y decir) y que revelan su significado originario (Galán Rodríguez 1998: 86), aunque estos no suelen presentar prácticamente variación en su forma (Bach Martorell 2009: 42). De hecho, este carácter menos gramaticalizado le concede un valor metalingüístico claro, relacionando formas distintas como parte de un hilo discursivo común (Galán Rodríguez 1998: 96). Otros marcadores que tienen como base el verbo decir también han resultado productivos en nuestro corpus como reformuladores, tal es el caso de digamos y digo (Ruiz-González en prensa), aunque en otras comunidades aparecen formas como vale decir (San Martín Núñez 2014; Garrido Martín 2015; Valencia Espinoza 2015), quiero decir (Guirado Zapata 2015; Hernández Cabrera 2015) o vamos a decir (González Mafud y Perdomo Carmona 2015).

Su orientación es, además, diferente a la que tiene lugar en o sea, que parte de un contenido hacia una forma o significante que engloba lo anteriormente expuesto, mientras es decir deriva, por lo general, de un significante hacia una definición (Galán Rodríguez 1998: 86), siendo que en ambos el interlocutor conoce una de las versiones del elemento que se plantea, pero desconoce la nueva que el hablante trata de explicar (Galán Rodríguez 1998: 97). Además, los elementos de esta locución adverbial constituyen un todo "que no puede desmembrarse ni modificarse" (Ciapuscio 2001: 162) y denotan cierta falta de compromiso en la enunciación, asumiendo el cariz de "es evidente" (Galán Rodríguez 1998: 99-100).

A veces, su aparición, que siempre es inicial de acto, puede ser opcional, especialmente cuando el grado de paráfrasis es casi total y es posible mover los miembros del discurso fácilmente (Garcés Gómez 2006: 175). Si aparece es por cuestiones de índole textual que facilitan el entendimiento del discurso, especialmente en textos escritos (Ciapuscio 2001: 169), así como en los casos en que expresa valores de corrección o conclusión, donde su presencia se vuelve casi obligada para una comprensión eficaz.

En la oralidad es, como ya hemos apuntado, menos frecuente que $o$ sea, pero de carácter más informal que otro de sus sinónimos, esto es (Martín Zorraquino y Portolés Lázaro 1999: 4123-4124; Martí Sánchez y Fernández Gómiz 2013: 78). En este contexto no presenta grandes variaciones, más allá de su reducción fonética a /é $\theta i R /$ o /ésiR/, según la variedad dialectal donde nos situemos (Holgado Lage 2017). 


\section{METODOLOGÍA}

Nuestro trabajo se integra dentro del Proyecto para el Estudio del Español de España y América (PRESEEA), un macroproyecto cuyo principal objetivo es construir, a través de una metodología común, un corpus sincrónico de las principales ciudades de España y América, que facilite el intercambio científico y la comparación de resultados en torno a una serie de temas (Moreno Fernández 1996: 258) desde todos los niveles de la lengua.

Las tres variables sociales que se han de tener en cuenta son: la edad, que diferencia tres generaciones, de 20 a 34 años, de 35 a 54, y de 55 en adelante, el sexo y el grado de instrucción, dentro del cual se distinguen tres subvariables: nivel de instrucción bajo (personas sin estudios o con estudios primarios), nivel de instrucción medio (personas con estudios secundarios) o nivel de instrucción alto (formación universitaria), tal como se expone en el cuadro 1.

\begin{tabular}{|l|c|c|c|c|c|c|c|}
\cline { 2 - 7 } \multicolumn{1}{c|}{} & \multicolumn{2}{c|}{$\begin{array}{c}\text { Generación 1 } \\
\text { (19-34 años) }\end{array}$} & \multicolumn{2}{c|}{\begin{tabular}{c} 
Generación 2 \\
\multicolumn{1}{c|}{$(\mathbf{3 5 - 5 4}$ años) }
\end{tabular}} & \multicolumn{2}{c|}{$\begin{array}{c}\text { Generación 3 } \\
\text { (más de 55 años) }\end{array}$} & \multirow{2}{*}{ Total } \\
\cline { 2 - 7 } & Hombres & Mujeres & Hombres & Mujeres & Hombres & Mujeres & \\
\hline $\begin{array}{l}\text { Nivel } \\
\text { alto }\end{array}$ & 3 & 3 & 3 & 3 & 3 & 3 & 18 \\
\hline $\begin{array}{l}\text { Nivel } \\
\text { medio }\end{array}$ & 3 & 3 & 3 & 3 & 3 & 3 & 18 \\
\hline $\begin{array}{l}\text { Nivel } \\
\text { bajo }\end{array}$ & 3 & 3 & 3 & 3 & 3 & 3 & 18 \\
\hline Total & 9 & 9 & 9 & 9 & 9 & 9 & 54 \\
\hline
\end{tabular}

Cuadro 1: Distribución de los informantes según las variables edad, sexo y nivel de instrucción

El cuestionario se aplicó tomando una muestra por cuotas de afijación uniforme, en la que la población se clasifica en estratos o categorías y se atribuye una cuota a cada uno de los distintos estratos (López Morales 1994: 58), asignando tres informantes para cada una de las celdas, lo que constituye un corpus de 54 informantes. Esta cifra, en una ciudad como Granada, con 237.929 habitantes en el momento de recogida de la muestra, supone un $0,0279 \%$, o, lo que es lo mismo, un informante por cada 4.406 habitantes (Moya Corral 2007: 43). 
Las grabaciones se llevaron a cabo entre 2005 y 2009 siguiendo los parámetros de PRESEEA, esto es, mediante un guion previo de preguntas organizadas alrededor de unos módulos temáticos concretos, con el que se persigue dar confianza al interlocutor para que se muestre lo más espontáneo posible (Moya Corral 2007: 44) y tratar de reducir así al mínimo la paradoja del observador (Labov 1972: 113). El resultado es un conjunto de conversaciones semidirigidas, o parcialmente dirigidas, sobre las que atenderemos únicamente los parlamentos de los informantes.

Después de un rastreo detallado de cada grabación y su correspondiente transcripción, se encontraron 48 realizaciones de es decir, que constituyen el objeto de análisis de este estudio. Su clasificación no resultó tarea fácil, al tratarse de una variable de tipo pragmático-discursiva, más aún con la dificultad añadida de la polifuncionalidad que caracteriza a los marcadores discursivos y que dificulta su reconocimiento y análisis (Poblete Bennett 1997: 74).

La concepción de una variable sociolingüística más allá del plano fonético ha tenido siempre una serie de complicaciones y cortapisas, especialmente tras el trabajo de Lavandera (1978), que puso en duda este tipo de estudios amparándose en la imposible equivalencia semántica entre variables. Sin embargo, según Moreno Fernández (2015 [1998]: 21), la variación lingüística, entendida como dos formas distintas de decir lo mismo, se produce en todos los niveles de la lengua, desde el fonético hasta el discursivo.

Para la detección del valor semántico de es decir en cada una de las ocurrencias, hemos aplicado la prueba de conmutación propuesta por Portolés Lázaro (2014 [1998]: 79-84). Así, por ejemplo, si el marcador puede intercambiarse fácil y preferentemente por esto es, consideraremos que su valor es explicativo, mientras que si es intercambiable por mejor dicho, en fin o así que, será rectificativo, recapitulativo o consecutivo, respectivamente. Aun así, no dejaremos de lado la relación semánticopragmática que se establece entre los miembros conectados por es decir y el grado de equivalencia entre ellos.

Para su tratamiento estadístico hemos utilizado el programa SPSS, en la versión 20 para Windows 10. El grado de significación lo hemos definido en el $5 \%$, según lo cual, si p valor es menor de 0,05 será estadísticamente significativo. Para saberlo llevamos a cabo la prueba paramétrica Análisis de varianza (ANOVA) y la no paramétrica ANOVA de Kruskal Wallis, previendo que la distribución de los datos pudiera ser anormal.

Además, puesto que la distribución puede venir condicionada por características de los hablantes externas a la lengua o por la duración media de cada una de las entrevistas, hemos ponderado los datos para reajustar el posible desequilibrio y que los resultados obtenidos fueran lo más fiables posible. 


\section{RESULTADOS}

\subsection{ANÁLisis CUALITATIVO}

Exactamente, como ya hemos indicado, hemos hallado 48 ocurrencias de la forma es decir en el corpus PRESEEA de la ciudad de Granada, lo cual, considerando que el total de reformuladores encontrados en dicha muestra es de 1186 (Ruiz-González 2020), supone un 4,05\% del uso total que se hace de este tipo de marcadores discursivos, una cifra bastante por encima de la arrojada en otras comunidades, como Santiago de Chile, donde apenas alcanzó un 0,87\% (San Martín Núñez 2017).

A la hora de estudiar cada uno de los casos, podemos percibir que se repiten con cierta frecuencia algunos sentidos propios o característicos de esta unidad. En primer lugar, la forma más común y prototípica de emplear este marcador es presentando tras él una unidad semejante -tanto semántica como pragmáticamente-a lo que se ha dicho de manera previa. Esto es, que tanto el miembro reformulado como el reformulador constituyan dos formas de decir lo mismo con otras palabras. Veamos este sentido en el ejemplo $1^{5}$.

(1) $\mathrm{I}^{6}$ : Bueno/ mira me gustaría/ por una parte/ al siguiendo con el tema del inglés/ me gustaría asentarlo pero/ $\mathrm{mm}$ yo me lo he toma(d)o en distintas fases// la primera fase// era// intentar que// un extranjero/ que un inglés me entendiera// hoy// creo que me entienden/ [aunque parezca un niño de dos tres años pero me da igual] es decir [aunque me puedan decir mira/ hablas como un niño de dos o tres años]// no me importa lo importante es que me entiendan un niño de de dos o tres años sabe ee/ por lo menos lo básico// y una vez que supero esta primera fase// mm me interesa muy mucho la pronunciación// mm quiero// voy a intentar hablar correctamente inglés// (GRAN-H23-097)

\footnotetext{
5 En cada ejemplo delimitaremos el primer miembro discursivo y el segundo entre corchetes y marcaremos el marcador en cursiva para que al lector le resulte más sencilla su visualización.

6 Abreviamos con la letra $i$ mayúscula el término informante, lo que denota que cada una de las palabras que aquí se exponen han sido recogidas de boca de los hablantes que conforman la muestra. En los casos en que se incluyan las palabras de la entrevistadora para comprender mejor el mensaje usaremos la letra e mayúscula.

7 Seguimos el sistema de codificación propuesto por PRESEEA y común a todos los equipos que lo forman. Según este, la ciudad se representa como GRAN (ciudad de Granada), en el sexo distinguimos $\mathrm{H}$ (Hombre) o M (Mujer); en la edad, 1 (Generación 1. ${ }^{\mathrm{a}}$ ), 2 (Generación
} 
Seguidamente, es frecuente también que es decir introduzca una definición más o menos amplia de un concepto o expresión ambiguos y que el hablante considere que el oyente puede llegar a interpretar de manera inadecuada, con lo cual introduce la reformulación para clarificar la situación comunicativa. Es el caso de los ejemplos 2 y 3.

(2) I: (...) en Psicología yo/ me centré mucho en lo que era la psicología experimental/y lo que era la/ (ruido = carraspeo) incluso hay una cosa que se llama psicología matemática y/ yo me centré mucho en esa parte y dentro de eso hay/ [toda una corriente// que/// mm que propugna lo que se llama el paradigma del procesamiento de información/] es decir [entiende//al/al hombre/ como// como objeto de estudio de la Psicología]/ como un canal de información//y entonces ee/ ¿qué utiliza qué medios utiliza// para estudiar ese canal de información? los mismos que para un canal de información como son los ordenadores (simultáneo: E = sí) (GRAN-H23-07).

(3) I: (...) y pero vaya ya estoy un poco de manera definitiva en el/ en el barrio del Albacín// que es un barrio ya digo tradicional// muy bonito/ de casas bajas/ de gente modesta// que/ ee tiene/ ee/ mucho jardín mucho jardín todas las casas ee... [lo típico de Granada que son los cármenes/] es decir [una casa que tiene/ como una especie de huerta//] y/ mi casa pues responde un poco a esa estructura/ mi casa está hecha un poco con el diseño// de la casa tradicional granadina// y además el Albaicín es un barrio que está así en pendiente por tanto// ee las casas/ pues a veces se acomodan al terreno que es lo que ocurre en mi casa// (GRAN-H33-013).

De hecho, en el segundo ejemplo, se observa cómo la conversación trata asuntos relativamente complejos para quien pueda no estar habituado al ámbito de la computación y la estadística, en este caso, y el emisor procura dar una explicación más sencilla o comprensible como forma de congraciarse con su interlocutor, tomando en cuenta que la máxima del uso de la reformulación, en cualquiera de los casos, es que el discurso sea interpretado de forma idónea.

En tercer lugar, puede ocurrir que la explicación se vea forzada por la situación comunicativa, en la que el hablante supone una serie de conocimientos implícitos en la conversación que no siempre se comparten

\footnotetext{
2. ${ }^{a}$ ) y 3 (Generación 3.a); y en el nivel de estudios encontramos 1 (Instrucción 1, nivel bajo), 2 (Instrucción 2, nivel medio) y 3 (Instrucción 3, nivel alto). Asimismo, cada informante recibe un número de la muestra; en el caso del primer ejemplo es el 09 porque se corresponde con el hablante número 9 .
} 
con el oyente. Así, el primero decide introducir un elemento reformulador que aclare adecuadamente sus intenciones en el discurso, ante cualquier gesto o mirada de incertidumbre suscitada por el segundo sobre ellas (ejemplo 4):

(4) I: Bueno/ pues fijate tú// $\mathrm{mm}$ es como si te te v(o)y a poner un ejemplo y es el tema de los albañiles// albañiles hay muchos// pero cualifica(d) os hay muy pocos// ¿por qué? porque realmente no hay gente que/ que forme a los albañiles entonces ¿cómo se aprende? pues// alguien te coge co(palabra cortada) coge al chavalillo lo pone allí con él y// [transcurrido un tiempo el chaval ya se/ se le da su título//] es decir [su título es que ya te/ él decide que puede//] o está prepara(d)o para para emprender su vida laboral como albañil/ por él solo por él solo ¿no?/ (GRAN-H23-09)

$\mathrm{Y}$ en otros casos lo que sucede es que se parte de una explicación vaga o confusa, en la que el propio hablante no tiene del todo claro lo que quiere decir y la reformulación le sirve de engarce hacia una premisa o un matiz, al que él mismo llega después de un rodeo, mientras piensa en lo que va a decir o busca los términos apropiados para hacerlo. Este cuarto valor puede apreciarse en el ejemplo 5.

(5) I: me gusta mucho/ tengo o sea llevo autobuses de gran calidad// ts me gusta mi profesión// lo que pasa que/ ya te digo/ hay algunos días/ (fragmento ininteligible) la presión/ la tensión que acumula el tráfico/ la propia gente también que te acumula/y hay días que los quieres olvidar/ pero reconozco que me gusta// y yo siempre/ ee/ yo siempre he pensado que/ ¡hombre!/ [en las profesiones se pueden le elegir]/ es decir/ [cuál es cualquier profesión]/ si no tienes otra cosa tienes que hacer/ pero/ si puedes elegir tu profesión/ para mí mejor/ (tiempo: 37:00) porque/ si tienes vocación/ bastante mejor evidentemente/ se hace con más iprofesionalidad!/ que si no tienes vocación/ que yo conozco gente/ que no tienen vocación de conductores/y/para mí son un poquito pésimos/y lo mismo en mi profesión/ que en miles profesionales yo creo/ por ejemplo// (GRAN-H22-025).

Del mismo modo, en quinto lugar, hallamos valores modalizadores del marcador. Por un lado, casos en los que el marcador mitiga o atenúa la información precedente y presenta una especie de rectificación al acto o actos previos, como en los ejemplos números 6 y 7. Es decir introduce en ellos un elemento que podríamos considerar equivalente a 'me refiero a' o 'esto es lo que quiero decir', que dispersa así cualquier posible duda suscitada en 
el interlocutor y salvaguarda la imagen del informante ante este ${ }^{8}$. En este caso es común que el acto previo haya quedado truncado.

(6) I: Ee más calida(d) musical// ¿eh?// más conocimiento// una mayor cultura musical/ general// mucho más que aquí// ee allí casi to(d)o el mundo/ [es raro quien no toca algún instrumento]// es decir [si toca algún instrumento es porque conoce el lenguaje musical ¿no?] porque/// entonces bue(palabra cortada)/ bueno si no todo el mundo a lo mejor me estoy pasando ¿no? pero/ ts hay como un eslabón/ un escalón más// más alto// culturalmente musical/ ee sobre música que aquí. (GRAN-H13-03)

(7) I: después te encuentras/ arriba// [lo que es el paseo el paseo Fe- mm Federico García Lorca// que le llaman// que se llama así]... es decir [vamos al paseo// vamos al paseo/ a pasear] (risas) porque el paseo es para pasear/ no tiene// no hay/ no hay ni entran motos ni entran coches ni entran bicis ¡vamos!// (tiempo: 49:02) bueno bicis algún crío que otro ¿no? pero que/ que el paseo con sus// con sus jardinitos en medio con sus fuentes/ en cada// ¿no?/// sus/ sus sus barillos de tapas// su quiosco de los helados/ sus/ el caño/ el pitorrillo// como lo llamaba mi abuelo el pitorrillo para beber agua/// y ya está y después está el hogar del pensionista// que está al fondo// y después/ sigues más arriba/// (GRAN-H12-020).

Y, por otro, usos intensificadores, pues el miembro reformulador sirve de realce de la idea anterior, pero con un matiz negativo o agravado de la situación que se venía planteando con anterioridad. Para entenderlo podemos observar el ejemplo número 8 .

(8) I: (...) descansar básicamente/ y cantar flamenco con los de allí del barrio que se llevaron la guitarra/// pf des-/ [desconectar básicamente] es decir / [fuera el trabajo/ fuera los móviles]/ hemos venido a descansar (GRAN-H11-037).

Por último, y relacionados con la combinación de es decir y la conjunción que, derivan los usos conclusivo y consecutivo. En el primero de los casos, se pretende recalcar la idea principal sobre una serie de hechos que se han enumerado de forma previa, especialmente en tipos de discurso donde el hablante expone su opinión abiertamente y pone en riesgo su imagen, o como

\footnotetext{
8 En estos casos, por tanto, podemos entender el empleo del marcador como una estrategia de cortesía positiva que refuerza las relaciones sociales entre los interlocutores. Se incluiría dentro de lo que Albelda Marco y Barros García (2013) llaman actos de refuerzo de la imagen. De hecho, en Albelda et al. (2014) se contempla la reformulación -a través de partículas u otros procedimientos- como un mecanismo usual de atenuación.
} 
resumen de un conjunto de hechos o cualidades introducidos con motivo de una descripción. En el segundo, lo que expresa el miembro reformulador es una consecuencia directa de una serie de hechos formulados previamente.

(9) I: estuve// que es uno de los más bonitos que yo he visto// en el Gran Capitán ¡la Vírgen! qué bonito era eso// otras veces he ido al/a Boñue-no a Bañuelos no/ al/ que hay allí en la Carrera del Darro frente a San Pedro $\mathrm{E}:$ los baños árabes

I: no/// el otro el Museo Arqueológico

E: ¡ah! Sí

I: [allí también// otras veces/ otras veces en el Corral del Carbón]/ es decir / [que/ que yo a todo eso me apunto]// luego he visto// de la Alhambra la he visto ya una pila de veces// porque también fui// porque ahora están dando en// en la Alhambra// los domingos por la tarde es gratis (GRAN-H31-049).

(10) I: ¿Mi método?/ la verdad es que yo soy un poco anárquico/o tal vez// soy muy autodidacta// entonces yo tengo// muchos problemas con el/con este método/ de entrada yo mi carrera la hice sin ir a clase// es decir [yo no he ido nunca a clase]/ es decir [te puedes imaginar/ qué método tengo ¿no?//] vamos que siempre lo único que yo hacía era/ ir a clase un día/ pero// en los descansillos/ para hablar con el delega(d)o de turno y decirle que yo estaba allí matricula(d)o y tal que si me podía dejar los apuntes o alguien que tuviera apuntes// na(da)/ o sea que yo me he dedica(d)o a traducir/ (GRAN-H23-09).

\subsection{ANÁLISIS CUANTITATIVO}

\subsubsection{Análisis cuantitativo bivariable}

Las ocurrencias sobre cuyos sentidos hemos hablado con anterioridad se reparten de la siguiente forma para cada una de las variables sociales tomadas en consideración:

\begin{tabular}{|c|c|c|c|c|c|c|c|}
\cline { 2 - 7 } \multicolumn{1}{c|}{} & \multicolumn{2}{c|}{$\begin{array}{c}\text { Generación 1 } \\
\text { (20-34 años) }\end{array}$} & \multicolumn{2}{c|}{$\begin{array}{c}\text { Generación 2 } \\
\mathbf{( 3 5 - 5 4} \text { años) }\end{array}$} & \multicolumn{2}{c|}{$\begin{array}{c}\text { Generación 3 } \\
\text { (más de 55 años) }\end{array}$} & \multirow{2}{*}{ Total } \\
\cline { 2 - 7 } \multicolumn{1}{c|}{} & Hombres & Mujeres & Hombres & Mujeres & Hombres & Mujeres & \\
\hline Nivel alto & 3 & 1 & 14 & 4 & 1 & 2 & 25 \\
\hline $\begin{array}{c}\text { Nivel } \\
\text { medio }\end{array}$ & 6 & 1 & 7 & 0 & 0 & 0 & 14 \\
\hline Nivel bajo & 1 & 1 & 0 & 0 & 7 & 0 & 9 \\
\hline Total & 10 & 3 & 21 & 4 & 8 & 2 & 48 \\
\hline
\end{tabular}

Cuadro 2: Resultados generales del uso de es decir según las variables sociales 
A pesar del limitado número de ocurrencias, a simple vista observamos en el cuadro 2 que esta partícula predomina en los hablantes con estudios superiores o universitarios, mientras se va reduciendo a medida que el nivel de instrucción disminuye. Además, es este grupo social el único en el que todos los hablantes emplean este marcador al menos una vez. Esto comprobaría nuestra teoría de que es decir es una unidad formal y culta, que conocen y utilizan con mayor frecuencia quienes han alcanzado un nivel educativo superior.

En cuanto a la edad, parece ser la segunda generación la que más lo utiliza, quizá porque es en este momento cuando los sujetos se hallan en pleno desarrollo profesional y emplean formas lingüísticas de la variedad "estándar" (Moreno Fernández 2015 [1998]: 50), que representen mejor esa situación de cara al resto de individuos con los que interaccionan. Además, los grupos de menor edad parecen decantarse por formas más propias de la oralidad espontánea (Santana Marrero 2014). Aun así, la escasez de datos hace que no sean del todo concluyentes.

No ocurre lo mismo en relación con la variable sexo, donde se observa un uso marcadamente elevado por parte de los hombres frente a las mujeres. Esto rompería un poco la tendencia a creer que las mujeres dudan más y se muestran más inseguras en sus argumentos, pues precisan con menor proporción volver sobre lo dicho previamente y matizar su discurso, como ocurre también en Cestero Mancera y Albelda Marco (2012) al analizar la atenuación lingüística, aunque sería necesario indagar cómo interviene este factor en el uso de otros mecanismos similares. Además, pese al conservadurismo que se ha asociado a las mujeres generalmente, en nuestro corpus ellas prefieren otras formas de carácter más coloquial, como vamos (Ruiz-González 2019).

Cuando entramos en el análisis estadístico inferencial, la varianza de ANOVA nos ayuda a comprobar que existe una dependencia entre el uso de es decir y el sexo de los hablantes granadinos, pues encontramos un Chi cuadrado de 17,817 y un $\mathrm{p}$ valor de 0,000 , que demuestra significatividad, aunque serían necesarios más datos para clarificar si los hombres emplean verdaderamente con mayor frecuencia esta partícula, tal como ocurría, por ejemplo, con o sea (Ruiz-González 2018) y como puede observarse de manera clara en el gráfico 1. Estos resultados no nos sorprenden si tenemos en cuenta, por ejemplo, los estudios sobre marcadores discursivos dentro del Proyecto de la norma culta (Valencia Espinoza 2014; Valencia Espinoza y Vigueras Ávila 2015), en los que, de manera general, son ellos quienes más emplean esta partícula, al menos en Buenos Aires, Caracas y México. También en el estudio de Taibo Cao (2016) para Montevideo son los hombres los que más lo utilizan. 


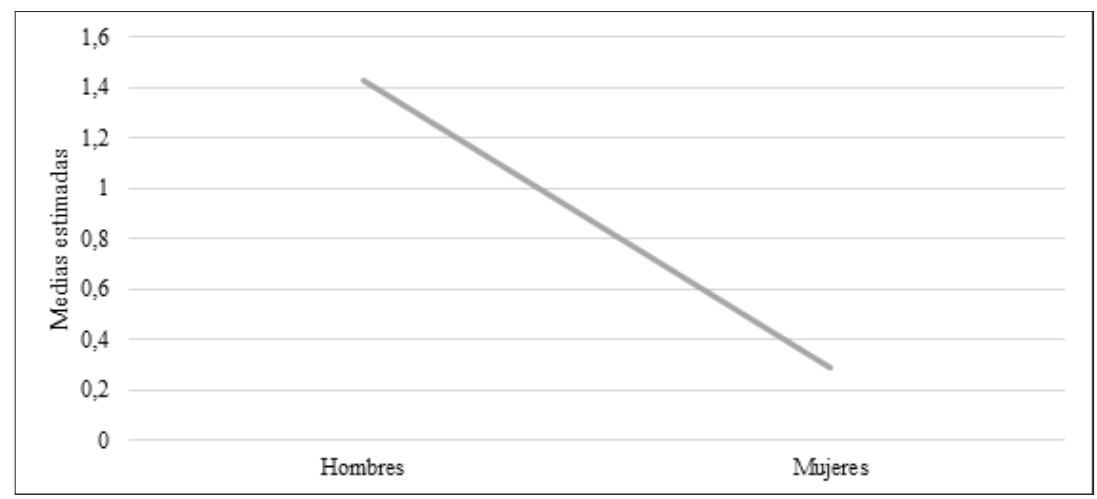

Gráfico 1: Medias estimadas del uso de es decir según el sexo de los hablantes granadinos

La prueba no paramétrica comprueba que, efectivamente, hay una dependencia entre el empleo de estas unidades y el sexo de los hablantes con datos como 9,264 para el Chi cuadrado y 0,002 para $p$ valor.

En cuanto a la variable social edad, los datos de Chi cuadrado y p valor en la varianza de ANOVA indican que su relación con respecto al uso de es decir es de dependencia, con unos resultados de 4,814 y 0,009 respectivamente, a favor, principalmente, de la segunda generación, como comprobamos en la representación gráfica número 2 .

La prueba de Anova de Kruskal Wallis, por su parte, validó dicha relación, pues obtuvimos unos datos de Chi cuadrado de 6,711 y un p valor de 0,035 .

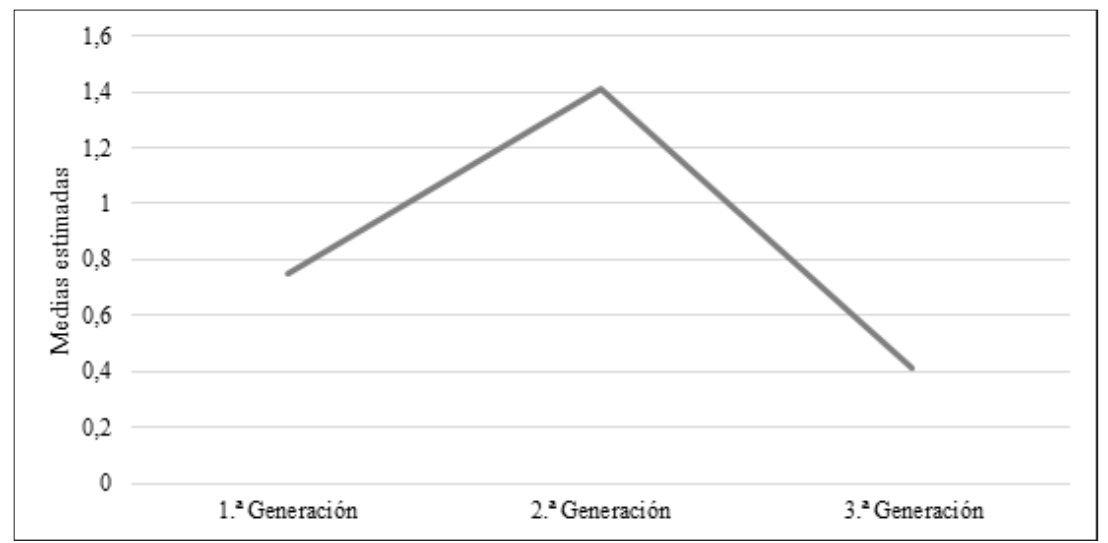

Gráfico 2: Medias estimadas del empleo de es decir según la edad de la muestra 
La comparación diacrónica entre muestras que se llevó a cabo en los trabajos relativos a la norma culta (Valencia Espinoza 2014; Valencia Espinoza y Vigueras Ávila 2015) determinaba que el uso de es decir por parte de los hablantes de primera generación estaba disminuyendo notablemente en las generaciones más jóvenes, donde es más frecuente $o s e a^{9}$. Ocurre igual en el nivel sociocultural alto de Montevideo, donde el $80,7 \%$ de los casos de es decir se corresponden con hablantes de tercera generación (Taibo Cao 2016), o en Santiago de Chile, donde el marcador directamente no aparece en el grupo etario más joven (San Martín Núñez 2017). En el análisis comparativo que efectúan Santana Marrero y Borzi (2020) se deja notar también una preferencia por el empleo de $o$ sea en el conjunto de hablantes de la ciudad de Sevilla, no tanto así en Buenos Aires, donde el empleo de es decir es mayor. Sin embargo, si rescatamos los datos de Borzi (2014, 2015) comprobamos que la muestra de 1960 revelaba un total de 120 casos de es decir frente a los 11 de 2012, esto es, una reducción de más del $90 \%$ en la frecuencia de empleo del marcador en apenas cincuenta años, mientras que en el caso de $o$ sea se pasa de 4 casos en el primer muestreo a 119 en el segundo, suponiendo un incremento del $97 \%{ }^{10}$. Sería, por tanto, interesante ampliar la muestra a grupos de edad inferior y comprobar si en Granada sucede algo similar, tal como revelan estos resultados.

En cuanto al nivel educativo -la variable que, en apariencia, tenía mayor relevancia en la manifestación de es decir-, la varianza de ANOVA revela que es realmente así, pues obtuvimos unos datos de Chi cuadrado de 16,522 y p valor de 0,000 , respectivamente, moviéndose el empleo de menos a más en cuanto inferior es el grado de instrucción de los hablantes. Así lo observamos en el gráfico 3.

\footnotetext{
Algunos autores consideran que hay un desplazamiento de es decir hacia o sea en los sociolectos de menor edad. Sin embargo, tal como ya dimos cuenta en un trabajo anterior sobre o sea (Ruiz-González 2018), en el corpus granadino el empleo de esta forma disminuye también en los hablantes jóvenes que emplean más asiduamente formas como hombre o vamos, de marcado carácter conversacional.

10 En Caracas también es significativo el aumento del empleo de o sea frente a es decir, que pasa el primero de 50 casos en 1973 a 127 en 2008 y el segundo, de 27 a 2 (Guirado 2015); o lo que es lo mismo, el empleo de $o$ sea aumenta un 72\% y el uso de es decir se reduce un $93 \%$. En La Habana, por su parte, el objeto de nuestro análisis pasa de 85 tokens en 1990 a 45 en 2010, frente a $o$ sea, que presenta 10 ocurrencias en la primera muestra frente a 80 que se documentan en la segunda (González Mafud y Perdomo Carmona 2015). Por tanto, en la ciudad cubana es decir se reduce un $35 \%$ mientras que o sea aumenta un $88,89 \%$. El resto de ciudades, en cambio, presentan un número de elementos poco determinante para concluir un cambio de tendencia.
} 
Con relación a la prueba no paramétrica, los datos de Chi cuadrado y $\mathrm{p}$ valor determinan la relación existente entre el mayor nivel de estudios y la aparición de es decir. Estos resultados fueron de 4,944 y 0,008, respectivamente.

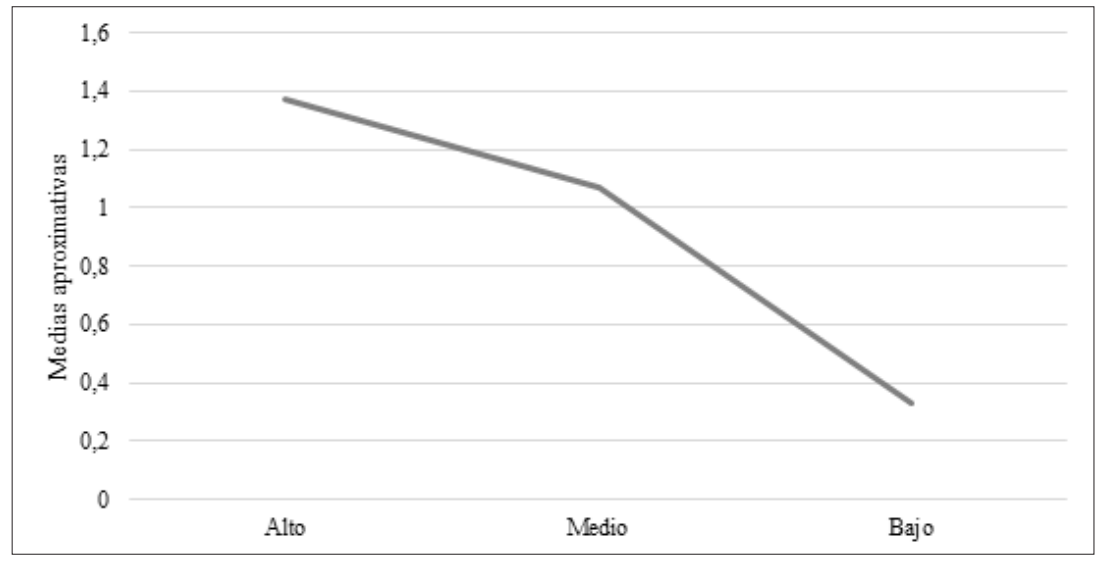

Gráfico 3: Medias aproximadas del uso de es decir en función del nivel educativo

Así lo comprobamos al contrastar los datos con otras ciudades, como la capital chilena, donde apenas se emplea en el nivel bajo (San Martín Núñez 2014, 2017) o Sevilla, donde este marcador presenta muy poca frecuencia en el estrato inferior en comparación con otros reformuladores, como o sea (Santana Marrero 2015b). Esto podría, por tanto, determinar que, en general, en español es decir es una unidad lingüística propia de los hablantes cultos.

\subsubsection{Análisis cuantitativo multivariable}

Según lo expuesto, cada una de las variables sociales tenidas en cuenta influyen o determinan de alguna manera la aparición de la forma es decir en el discurso de los hablantes de la muestra. Pero, como nuestra intención es conocer en profundidad cómo se produce la elección de este marcador y no otro para reformular, hemos decidido cruzar las variables diastráticas entre sí para comprobar qué sucede. Los resultados hallados se muestran a continuación.

En primer lugar, observamos en el gráfico 4 la relación entre la edad y el sexo y la frecuencia de uso de la unidad estudiada. Vemos que, en general, el empleo de la forma es decir disminuye en las mujeres con respecto a los hombres, siendo especialmente significativo en la segunda generación, que es la que emplea con mayor frecuencia el reformulador. En las otras dos también son las féminas las que lo emplean menos, pero no hay un contraste tan obvio. 


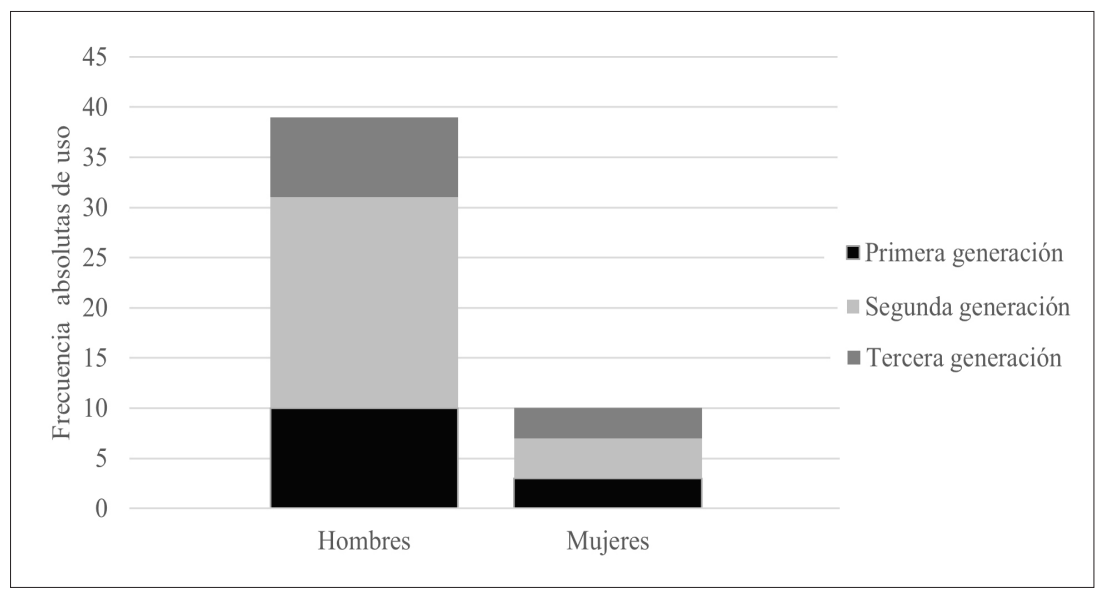

Gráfico 4: Frecuencias absolutas de es decir según el sexo y la edad de los hablantes

Elaboramos en el análisis una tabla de contingencia con ambas variables para observar si la relación entre la edad y sexo era determinante para que se diera el uso de es decir. La prueba de Chi cuadrado de Pearson ${ }^{11}$ arrojó un dato de 0,294 y una significación asintótica o $\mathrm{p}$ valor de 0,863 , con lo cual revela una no dependencia entre las dos variables estudiadas.

En el gráfico 5, por otra parte, observamos la distribución de las frecuencias absolutas de es decir según el sexo y el nivel de instrucción. En apariencia, se observa cómo, de forma general, el empleo del marcador discursivo por parte de las mujeres disminuye en los tres niveles educativos, siendo especialmente relevante en el nivel alto, pues es el que más lo utiliza, aunque también es notorio el caso de las féminas de nivel bajo que prácticamente no lo emplean.

11 La prueba $\mathrm{Chi}^{2}$ de Pearson es una prueba que mide la diferencia entre una distribución observada y la esperada y sirve para probar la dependencia o la independencia de dos variables entre sí (Herrera Soler, Martínez Arias y Amengual 2011: 183). 


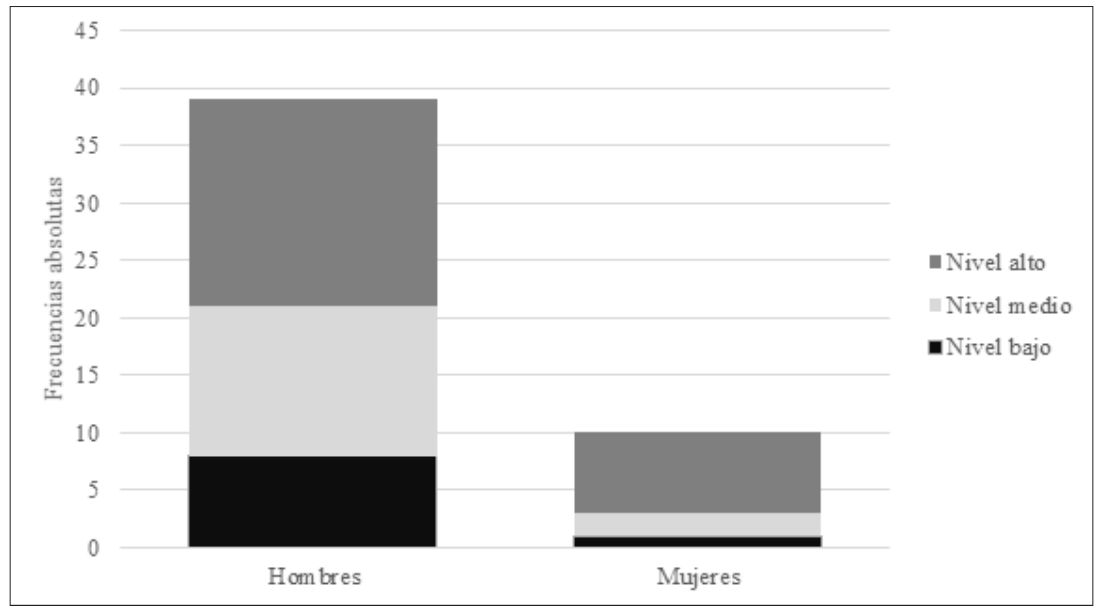

Gráfico 5: Valores absolutos de es decir en función del sexo y el nivel de instrucción

La prueba de Chi cuadrado a la que antes aludíamos muestra que no existe dependencia entre ambas variables, puesto que los resultados de $\mathrm{Chi}^{2}$ y $\mathrm{p}$ valor fueron de 2,987 y 0,225 , respectivamente.

Por último, en cuanto a la relación entre el nivel de instrucción y la edad, los resultados que se reflejan en el gráfico 6 sí presentan ciertos cambios con relación a las variables anteriores.

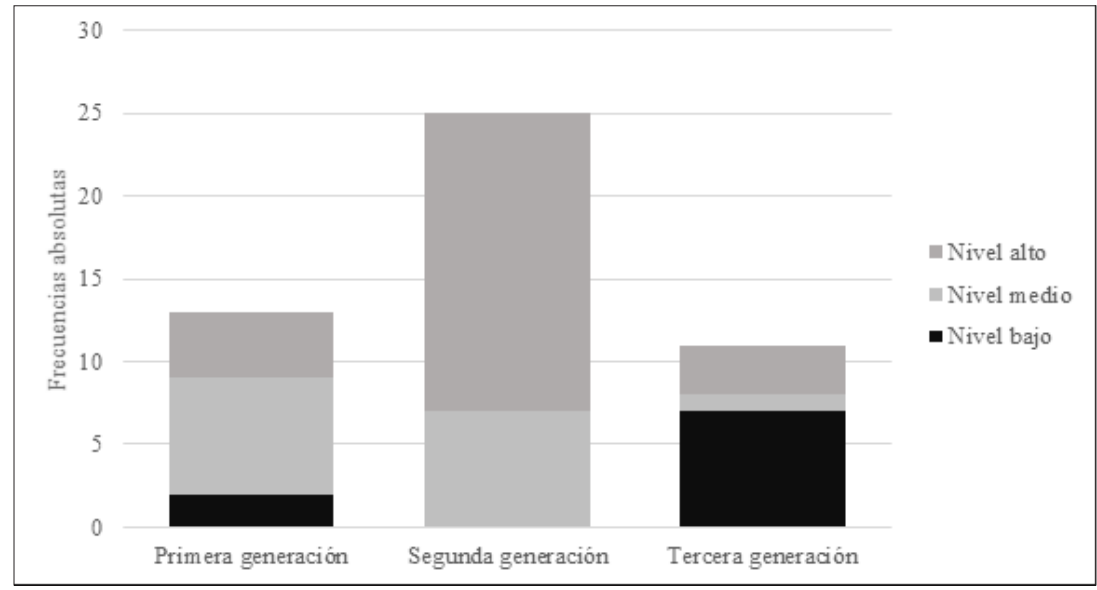

Gráfico 6: Frecuencias absolutas de la forma es decir con relación a la edad y el nivel educativo 
En primer lugar, observamos que hay un pico de uso en el grupo etario de segunda generación del nivel alto, muy por encima del empleo que jóvenes y mayores de este nivel llevan a cabo. Esto podría deberse a que son precisamente los miembros de este grupo los que se insertan dentro del mercado laboral y lidian, a menudo, con una terminología poco usual para otro tipo de público. De ahí la necesidad de explicar de forma continua lo que han pretendido comunicar.

En segundo término, llama la atención que, en el nivel medio, aunque se usa con poca frecuencia, son los hablantes jóvenes y de mediana edad quienes más lo utilizan, frente a los hablantes de tercer grupo etario, que prescinden casi por completo de él. Creemos que esto se debe a que las dos primeras generaciones se hallan inmersas en el mundo laboral y buscando su propio espacio y lugar en la sociedad de la que forman parte. Por ello recurren a fórmulas que son propias del nivel educativo alto, a modo de hipercorrección, clásica de las clases medias que tratan de adaptarse e imitar en su modo de hablar a los hablantes de nivel culto o superior (Labov 1970: 287).

En el nivel bajo, por su parte, son los hablantes mayores los que hacen un uso destacado de es decir frente a sus iguales. Esto puede deberse a que, llegados a una edad de madurez considerable, las relaciones sociales cambian y se equilibran de alguna forma. Las personas con un cierto recorrido, que solo concede el transcurrir de los años y las experiencias, pueden llegar a percibir mejor la necesidad de que el discurso sea lo más claro y preciso posible para su correcta interpretación, con independencia de su nivel de estudios. Además, coincide con este grupo etario, principalmente, el uso reiterado de es decir con sentido conclusivo.

Las tablas de contingencia revelan que sí hay dependencia entre las variables sociales consideradas y la frecuencia de uso de es decir, ya que el $\mathrm{Chi}^{2}$ resultó de 28,392 y el p valor, 0,000 .

\section{CONCLUSIONES}

En relación con el empleo de la unidad de reformulación es decir en el español de Granada y su variación sociopragmática, exponemos las siguientes conclusiones, referidas a las funciones de este marcador y la variación observada.

Con respecto a los valores que toma es decir, podemos destacar que, aunque su uso es principalmente explicativo, toma diferentes variantes 
de sentido con relación al contexto en el que se integra. Así, hemos encontrado que puede servir para presentar unidades equivalentes semántica y pragmáticamente, para definir un concepto complejo o una idea que ha aparecido de forma vaga o ambigua, al tiempo que mitiga cierta información y rectifica ante la forma de expresar un determinado asunto en el que el hablante puede ver dañada su imagen, sobre todo en actos de carácter argumentativo o de opinión. También se utiliza para concluir o mostrar la consecuencia de una serie de hechos enumerados con anterioridad y que el hablante pretende condensar en un único miembro discursivo que facilite la comprensión de su interlocutor, máxima que lo guía continuamente para emplear este tipo de marcadores en la conversación.

En cuanto a su variación sociolingüística, hemos comprobado, en primer lugar, que es el nivel alto el que usa predominantemente esta partícula. En segundo lugar, parece no cumplirse la hipótesis de que sean los hablantes de tercera generación quienes la prefieran, pues es el segundo grupo de edad el que la utiliza principalmente. Los miembros de este último grupo etario se hallan inmersos en el mercado laboral y buscan construir su imagen social ante el resto de interlocutores con los que se relacionan. Esto supone el uso de formas tradicionalmente entendidas como formales, tal como ocurre con es decir, cuyo uso supone, para los hablantes de niveles educativos inferiores, una hipercorrección o imitación de lo que consideran culto. En tercer lugar, llama la atención que el sexo se revele como una variable extralingüística determinante para la aparición del reformulador: el uso de es decir es claramente mayoritario en los hombres, mientras que las mujeres, en teoría más conservadoras, prefieren usar otras formas menos gramaticalizadas y más coloquiales, como vamos.

Con vistas a trabajos futuros, consideramos que sería interesante ampliar la pequeña comparación que hemos querido formular con respecto a las frecuencias de uso de es decir y las variables sociales tomadas en cuenta, especialmente sobre los corpus PRESEEA que ya han tratado de una u otra forma el empleo de estas unidades, y comprobar si, como podemos prever, existe un cambio de tendencia hacia la pérdida de es decir en las generaciones más jóvenes a favor de o sea. Asimismo, nos interesa también conocer cómo el marcador se relaciona con otro tipo de variables y cómo estas determinan su aparición en la conversación, tal como el modelo de acto discursivo donde tiene lugar, ya que parece propenso a aparecer o bien con argumentaciones, o bien con descripciones, y el entorno sintáctico que lo acompaña, especialmente cuando va seguido de la conjunción que, que presenta matices recapitulativos y es frecuente hallarla con otros marcadores de reformulación como o sea o vamos. 


\section{REFERENCIAS BIBLIOGRÁFICAS}

Albelda Marco, Marta y María Jesús Barros García. 2013. La cortesía en la comunicación. Madrid: Arco Libros.

Albelda Marco, Marta, Antonio Briz Gómez, Ana María Cestero Mancera, Dorota Kotwica y Cristina Villalba IbáÑEz. 2014. Ficha metodológica para el análisis pragmático de la atenuación en corpus discursivos del español (Es.Por.Atenuación). Oralia: Análisis del discurso oral 17: 7-62.

Bach Martorell, Carme. 2009. La reformulación del discurso en español en comparación con el catalán. Estudio contrastivo de los marcadores de reformulación. En María Pilar Garcés Gómez (dir.). La reformulación del discurso en español en comparación con otras lenguas (catalán, francés, italiano, inglés, alemán e islandés), pp. 37-66. Madrid: Universidad Carlos III: Boletín Oficial del Estado.

Beinhauer, Werner. 1978 [1963]. El español coloquial. Madrid: Gredos.

Borzi, Claudia. 2014. Marcadores del discurso de Buenos Aires. En Alba Valencia Espinoza (ed.). Marcadores discursivos en la norma culta hispánica (1964-2014). Cuadernos de la ALFAL 5: 13-42. Disponible en http://www.mundoalfal.org/sites/default/files/ revista/05_cuaderno_002.pdf [Consulta 10/01/2021].

2015. Marcadores del discurso de Buenos Aires. En Alba Valencia Espinoza y Alejandra Vigueras Ávila (eds.). Marcadores hispánicos. Usos de España y América en el corpus de estudio de la norma culta, pp. 19-60. México: UNAM.

Briz Gómez, ANTONIO. 1997. El papel metadiscursivo del conector pragmático en español coloquial. En Beatriz Gallardo Paúls, Daniel Jorques Jiménez, María Amparo Alcina Caudet, Montserrat Veyrat Rigat y Enric Serra Alegre (coords.). Panorama de la investigació lingüistica a l'Estat espanyol: Actes del I congrés de lingüística general, vol. 3, pp. 69-77. Valencia: Universidad de Valencia.

Carbonero Cano, Pedro y Juana Santana Marrero. 2010. Los marcadores y la variación espacial y social. En Óscar Loureda Lamas y Esperanza Acín Villa (coords.). Los estudios sobre marcadores del discurso del español, hoy, pp. 497-521. Madrid: Arco Libros.

Casado Velarde, Manuel. 1991. Los operadores discursivos es decir, esto es, o sea y a saber en español actual: valores de lengua y funciones textuales. Lingüística Española Actual 13: 87-116.

Cestero Mancera, Ana María y Marta Albelda Marco. 2012. La atenuación lingüística como fenómeno variable. Oralia: Análisis del discurso oral 15: 77-124.

Ciapuscio, Guiomar E. 2001. Los conectores reformulativos: el caso de es decir. En Elvira N. De Arnoux y Ángela Di Tullio (coords.). Homenaje a Ofelia Kovacci, pp. 157-172. Buenos Aires: Eudeba. Disponible en https://dialnet.unirioja.es/servlet/articulo? codigo=900255 [Consulta 25/10/2019].

CorTÉs Rodríguez, Luis. 2008 [1998]. Marcadores del discurso y análisis cuantitativo. En María Antonia Martín Zorraquino y Estrella Montolío Durán (eds.). Los marcadores del discurso. Teoría y análisis, pp. 143-160. Madrid: Arco Libros.

Fuentes Rodríguez, Catalina. 1990. Procedimientos intradiscursivos: decir y los explicativos. En Pedro Carbonero Cano, Pedro y María Teresa Palet Plaja (eds.). Habla de Sevilla y hablas americanas. Colección Sociolingüistica Andaluza 5: 103-123. Sevilla: Publicaciones de la Universidad.

2009. Diccionario de conectores y operadores del español. Madrid: Arco Libros.

GALÁN RodríGuEz, CARMEN. 1998. La dimensión explicativa y deóntica de los conectores $o$ sea y es decir. Anuario de estudios filológicos 21: 85-104. Disponible en https://dialnet. unirioja.es/servlet/articulo?codigo $=58923$ [Consulta 07/11/2019] 
Garcés Gómez, María Pilar. 2006. El concepto de reformulación. En Juan de Dios Luque Durán (ed.). Actas del V Congreso andaluz de lingüistica general: Homenaje al profesor José Andrés de Molina Redondo, vol. 1, pp. 169-18. Granada: Método Ediciones.

Garrido Martín, Blanca. 2015. La historia de vale decir como un marcador discursivo de reformulación. Cahiers d'études hispaniques médiévales 38: 187-206. Disponible en https://dialnet.unirioja.es/servlet/articulo?codigo=5388488 [Consulta 08/01/2021].

González Mafud, Ana y Marialys Perdomo Carmona. 2015. Marcadores discursivos de La Habana. En Alba Valencia Espinoza y Alejandra Vigueras Ávila (eds.). Marcadores hispánicos. Usos de España y América en el corpus de estudio de la norma culta, pp. 207-255. México: UNAM.

2018. Aproximación al estudio de los marcadores discursivos en muestras de habla culta de La Habana. Universidad de La Habana 285: 25-49. Disponible en http:// scielo.sld.cu/scielo.php?script=sci_arttext\&pid=S0253-92762018000100002 [Consulta 12/03/2020].

Guirado Zapata, Kristel. 2015. Marcadores del discurso de Caracas. En Alba Valencia Espinoza y Alejandra Vigueras Ávila (eds.). Marcadores hispánicos. Usos de España y América en el corpus de estudio de la norma culta, pp. 69-122. México: UNAM.

2019. Reformulación locutiva/ilocutiva: o sea y es decir en una microdiacronía de la oralidad caraqueña. Ponencia presentada en el Discourse Markers in Romance Language 6, Bérgamo, Italia, 29-31 de mayo.

Hernández Cabrera, Clara. 2015. Marcadores del discurso de Las Palmas de Gran Canaria. En Alba Valencia Espinoza y Alejandra Vigueras Ávila (eds.). Marcadores hispánicos. Usos de España y América en el corpus de estudio de la norma culta, pp. 257-302. México: UNAM.

Herrera Soler, Honesto, María del Rosario Martínez Arias y Marian Amengual Pizarro. 2011. Estadística aplicada a la investigación lingüística. Madrid: EOS.

Holgado Lage, Anais. 2017. Diccionario de marcadores discursivos para estudiantes de español como segunda lengua. New York: Peter Lang.

JASKot, MACIEJ. 2012. Marcadores del discurso en español y esperanto. Breve estudio contrastivo con ejemplos del corpus del español hablado en Granada. En Juan Antonio Moya Corral y Edytta Waluch-De La Torre (eds.). Español hablado. Estudios sobre el corpus PRESEEA-Granada, pp. 25-36. Varsovia: Instituto de Estudios Ibéricos e Iberoamericanos de la Universidad de Varsovia.

Labov, William. 1970. The study of Language in its Social Context. Studium Generale 23: $30-87$.

1972. Some principles of Linguistic Methodogy. Language in Society 1: 97-120. 1983. Modelos sociolingüísticos. Madrid: Cátedra.

LAVANDERA, BEATRIz. 1978. Where does the sociolinguistic variable stop? Language in Society 7: 171-182.

López Alonso, Covadonga. 1990. El discurso y el conector reformulativo: es decir. Revista de filología románica 7: 87-100. Disponible en https://revistas.ucm.es/index.php/RFRM/ article/download/RFRM9090110087A/12840 [Consulta 12/12/2019].

López Morales, Humberto. 1994. Métodos de investigación lingüistica. Salamanca: Colegio de España.

Martí Sánchez, Manuel y Sara Fernández Gómiz. 2013. Los marcadores discursivos. Para estudiantes y profesores. Español como lengua extranjera. Madrid: Edinumen.

Martín Zorraquino, María Antonia y José Portolés Lázaro. 1999. Los marcadores del discurso. En Ignacio Bosque y Violeta Demonte (coords.). Gramática descriptiva de la lengua española, vol. 3, pp. 4051-4214. Madrid: Espasa Calpe. 
Montolío Durán, Estrella. 2018. Mecanismos de cohesión (II). Los conectores. En Estrella Montolío Durán (dir.). Manual de escritura académica y profesional. Estrategias gramaticales y discursivas, pp. 477-560. Barcelona: Ariel.

Montoro del Arco, Esteban T. 2011. Condicionamientos sociolingüísticos de la variación fraseoléxica: los operadores de foco de exclusión. Lingüística Española Actual 33, 2: 223-263.

2012. ¿Son los hablantes andaluces más expresivos? La variación pragmática en el uso de los operadores focales de exclusión en Granada y Valencia. En Juan Antonio Moya Corral y Edyta Waluch-de la Torre (eds.). Español hablado. Estudios sobre el corpus PRESEEA-Granada, pp. 77-93. Varsovia: Instituto de Estudios Ibéricos e Iberoamericanos de la Universidad de Varsovia.

Moreno Fernández, Francisco. 1996. Metodología del "Proyecto para el Estudio Sociolingüística del Español de España y de América" (PRESEEA). Lingüística 8: 257-287. Ariel.

2015 [1998]. Principios de Sociolingüística y Sociología del lenguaje. Barcelona:

Moya Corral, Juan Antonio. coord. 2007. El español hablado en Granada. Corpus oral para su estudio sociolingüístico (Nivel de estudios alto). Granada: Editorial Universidad de Granada.

Murillo Ornat, Silvia. 2016. Sobre la reformulación y sus marcadores. Cuadernos AISPI 8: 237-258. Disponible en https://dialnet.unirioja.es/descarga/articulo/6249639.pdf [Consulta 15/12/2019].

Poblete Bennet, María Teresa. 1997. Los marcadores discursivo-conversacionales en la construcción del texto oral. Onomázein 2: 67-81. Disponible en http://onomazein.letras. uc.cl/Articulos/2/2_Poblete.pdf [Consulta 25/03/2020].

PortolÉs LÁzaró, José. 2004. Pragmática para hispanistas. Madrid: Síntesis.

2014 [1998]. Marcadores del discurso. Barcelona: Ariel.

Real Academia Española. 2020. [DLE] Diccionario de la lengua española. Disponible en www.dle.rae.es [Consulta 10/01/2021].

REPEDE, DoINA. 2020. La construcción en plan en el español hablado de Sevilla: uso, función y distribución social. Tonos digital 38: 1-23. Disponible en http://www.tonosdigital.es/ ojs/index.php/tonos/article/view/2415/1135 [Consulta 28/12/2020].

Rodríguez Ramalle, Teresa M. 2014. Una nueva reflexión en torno a los marcadores de reformulación con que. Revista de Investigación Lingüística 17: 119-145. Disponible en https://dialnet.unirioja.es/servlet/articulo?codigo $=5014938$ \&orden $=1 \&$ info $=1$ ink [Consulta 17/11/2019].

Ruiz-GonzÁlez, Natalia. 2018. La reformulación discursiva en español: el caso de o sea. Itinerarios: Revista de estudios lingüísticos, literarios, históricos y antropológicos 28 : 177-199. Disponible en http://itinerarios.uw.edu.pl/la-reformulacion-discursiva-en-elespanol-de-granada-el-caso-de-o-sea/ [Consulta 15/03/2020]

2019. Los verbos de movimiento con valor reformulativo: el caso de vamos y vaya en el español de Granada. Ponencia presentada en el XXXIV Congreso Internacional de la Asociación de Jóvenes Lingüistas (AJL), Valencia, España, 18-20 de septiembre.

2020. Los reformuladores de recapitulación en el corpus PRESEEA de Granada. ELUA: Estudios de Lingüística de la Universidad de Alicante 34: 193-212. Disponible en https://revistaelua.ua.es/article/view/15360 [Consulta 27/12/2020].

en prensa. La estratificación social de los reformuladores en el español de Granada.

SAn Martín NuÑez, Abelardo. 2014. Variación sintáctica y discursiva en el español hablado en Santiago de Chile: análisis sociolingüistico del queísmo, el dequeísmo, el discurso referido y los marcadores de reformulación. Tesis doctoral, Valladolid, 
Universidad de Valladolid. Disponible en http://uvadoc.uva.es/handle/10324/15865 [Consulta 07/02/2020].

2016. Los marcadores de reformulación en el español oral de Santiago de Chile: análisis discursivo y sociolingüístico. Oralia: Análisis del discurso oral 19: 281-322.

2017. Análisis sociolingüístico de los reformuladores de explicación en el español hablado de Santiago de Chile. Revista Signos 50 (93): 124-147. Disponible en https://www. redalyc.org/pdf/1570/Resumenes/Resumen_157050381006_1.pdf [Consulta 27/02/2020].

San Martín NúÑEZ, Abelardo y Silvana Guerrero GonzÁlez. 2016. Los marcadores de reformulación en el corpus PRESEEA de Santiago de Chile. Forma y función 29, 1: 15-38. Disponible en https://revistas.unal.edu.co/index.php/formayfuncion/article/ download/60187/60252 [Consulta 15/03/2020].

Santana Marrero, Juana. 2014. Marcadores del discurso de Sevilla. En Alba Valencia Espinoza (ed.). Marcadores Discursivos en la Norma Culta Hispánica (1964-2014). Cuadernos de la ALFAL vol. 5, pp. 277-311. Disponible en https://www.mundoalfal.org/ sites/default/files/revista/05_cuaderno_010.pdf [Consulta 15/10/2019].

2015a. Luego and después with a textual function: discursive use and variation in the Panhispanic educated norm. Revista de Lenguas para Fines Especificos 21 (1): 160-183. Disponible en https://ojsspdc.ulpgc.es/ojs/index.php/LFE/article/view/244 [Consulta 07/01/2021].

2015b. Marcadores del discurso y variación diastrática: análisis en el sociolecto bajo. En Juana Santana Marrero (dir.), Marta León-Castro y Adamantia Zerva (eds.). La variación en el español actual. Estudios dedicados al profesor Pedro Carbonero. Colección Sociolingüística Andaluza 17, pp. 289-313. Sevilla: Universidad de Sevilla.

2015c. Marcadores discursivos de Sevilla. En Alba Valencia Espinoza y Alejandra Vigueras Ávila (coords.). Más sobre marcadores hispánicos. Usos de España y América en el corpus de estudio de la norma culta, pp. 443-490. México D.F.: UNAM.

2016. Luego y después: uso discursivo y variación. RILCE. Revista de Filología Hispánica 32 (2): 513-535.

2017. Marcadores interrogativos de interacción conversacional en la norma culta hispánica. 90 años de la Academia Boliviana de la Lengua, pp. 232-286. La Paz: Academia Boliviana de la Lengua.

2019a. Apéndices interrogativos en el habla de Sevilla: un estudio en tiempo real. Itinerarios: Revista de estudios lingüísticos, literarios, históricos y antropológicos 29: 297-318. Disponible en http://itinerarios.uw.edu.pl/wpcontent/uploads/2019/09/13 articulo_Santana_Marrero.pdf [Consulta 30/12/2020].

2019b. Los apéndices interrogativos en el corpus PRESEEA-Sevilla: sociolectos alto y bajo. Oralia: Análisis del Discurso Oral 22 (1): 111-127.

Santana Marrero, Juana y Claudia Borzi. 2020. Marcadores del discurso en la norma culta de Buenos Aires y de Sevilla: estudio contrastivo. Philologica Canariensia 26: 5679. Disponible en https://ojsspdc.ulpgc.es/ojs/index.php/PhilCan [Consulta 28/12/2020].

SAntos Río, Luis. 2003. Diccionario de partículas. Salamanca: Luso-Española.

Silva-Corvalán, Carmen y Andrés Enrique-Arias. 2017 [2001]. Sociolingüística y Pragmática del español. Washington D.C: Georgetown University Press.

Taibo CaO, Luis. 2016. Los marcadores del discurso en el habla culta de Montevideo: análisis del corpus PRESEEA. Trabajo final de máster. Madrid, Universidad Nacional de Educación a Distancia.

Valencia Espinoza, Alba. ed. 2014. Marcadores discursivos en la norma culta hispánica (1964-2014). Cuadernos de ALFAL, vol. 5. En línea: <https://mundoalfal.org/es/content/ cuadernos-de-la-alfal-n\%C2\%BA5> [Consulta 20/09/2019]. 
2015. Marcadores del discurso de Santiago de Chile. En Alba Valencia Espinoza y Alejandra Vigueras Ávila (eds.). Marcadores hispánicos. Usos de España y América en el corpus de estudio de la norma culta, pp. 377-441. México: UNAM.

Valencia Espinoza, Alba y Alejandra Vigueras Ávila. eds. 2015. Marcadores hispánicos. Usos de España y América en el corpus de estudio de la norma culta. México: UNAM.

Vigara Tauste, Ana María. 1992. Morfosintaxis del español coloquial: esbozo estilístico. Madrid: Gredos. 